\title{
Posterior mediastinal paraganglioma presenting with hypertension and back pain in a young adult
}

\author{
Tayfun Kermenli ${ }^{1}$, Cebrail Azar ${ }^{2}$ \\ ${ }^{1}$ Thoracic Surgery Clinic, Medicalpark Elazığ Hospital, Elazığ, Turkey \\ ${ }^{2}$ Chest Diseases Clinic, Medicalpark Elazığ Hospital, Elazığ, Turkey
}

Kardiochirurgia i Torakochirurgia Polska 2019; 16 (1): 47-48

Paragangliomas are rare neuroendocrine tumors that secrete neuropeptide and catecholamine. The majority of these tumors are benign, while approximately $10 \%$ are malignant. In the thoracic cavity they usually occur in the aorta-sympathetic area and posterior mediastinum. Functional paragangliomas present with paroxysmal hypertension, palpitation, and headache due to increased catecholamines [1]. Nonfunctional ones are usually postoperatively diagnosed and they have mediastinal, pulmonary or endobronchial origin [2]. As in our case, it can be present with hypertension in a young adult. We wanted to present this case because it is a rare case in terms of thoracic surgeons and the procedure was performed with a minimally invasive surgical technique.

A 24-year-old male patient had a history of arterial hypertension and tachycardia for the first time at the age of 20. The highest blood pressure of the patient was 180/90 and the pulse rate was 118 per minute. He was diagnosed with hypertension attacks, back pain, tachycardia and headache. He had a urine vanillylmandelic acid (VMA) level of $14.7 \mathrm{mg} / 24$ hours due to pheochromocytoma. Contrast- enhanced chest MRI showed a $3.8 \times 2 \mathrm{~mm}$ diameter, high vascularized and contrasted mass in the left para-aortic area at the end of the diaphragm (Fig. 1). Positron emission tomography-computed tomography (PET-CT) and metaiodobenzylguanidine (MIBG) scintigraphy were performed at an external center and they did not show active involvement in the mass. There was no maximum standard uptake (SUVmax) value. No areas of involvement in other parts of the body were detected.

The operation was planned with the patient considering paraganglioma based on these results. Carvedilol (12.5 mg, $1 \times 1$, p.o.) treatment was started for the patient to control hypertension by following cardiology recommendations. Following 1 week of antihypertensive treatment, preoperative preparations were completed and the patient was excised with video-assisted thoracoscopic surgery (VATS) and excision was performed. During the operation, esmolol i.v. solution was available for hypertension and tachycardia, but hypertension and tachycardia were not observed. For the resection, $1 \mathrm{~cm}$ incisions were opened on the $4^{\text {th }}$ and $7^{\text {th }}$ intercostal spaces (ICS) anterior axillary line and $8^{\text {th }}$ ICS
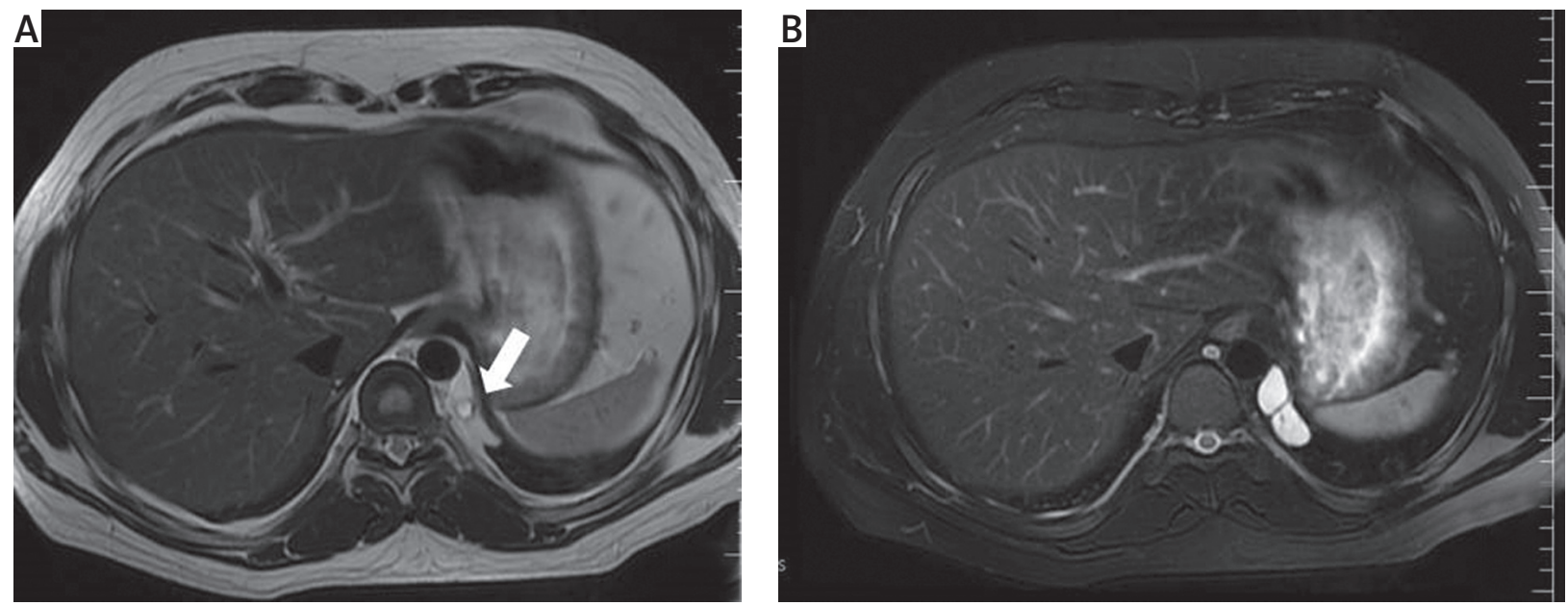

Fig. 1. The thorax magnetic resonance showing the mass with central necrosis in the left para-aortic area (arrow) (A), and contrast enhancement in the mass after contrast medium administration (B)

Address for correspondence: Tayfun Kermenli MD, Thoracic Surgery Clinic, Medicalpark Elazığ Hospital, 23700 Elazığ, Turkey, phone: +90 4242348000, e-mail: tayfunkermenli@gmail.com

Received: 12.11.2018, accepted: 5.01.2019. 


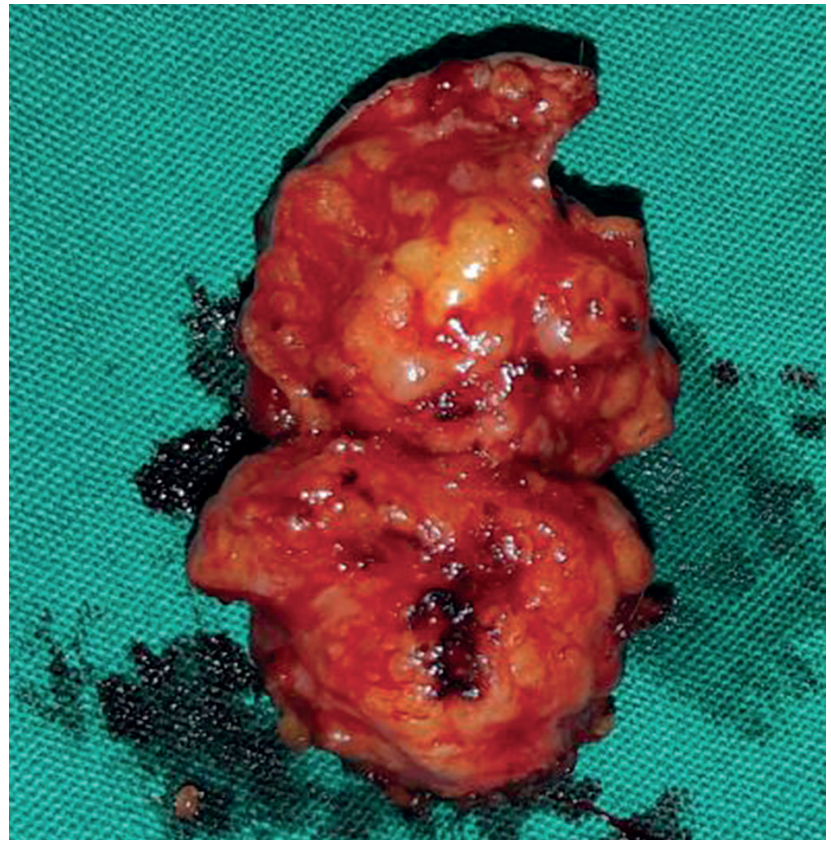

Fig. 2. Post-resection image of the mass

posterior axillary line. The mass was dissected from the surrounding tissues using endo-cautery. The main artery of the mass was $1 \mathrm{~mm}$ in diameter and it was clipped with the hemolock clip. Bleeding control was performed with endo cautery. After the resection, the mass was removed from the thorax with the endobag.

Paragangliomas have rich vascularization and they are hemorrhagic tumors; for this reason a hemovac drain $(10 \mathrm{Fr})$ was placed in the pleural cavity. Active drainage was not detected in the days following surgery. The hemovac drain was removed on the postoperative second day and the patient was discharged on the same day. Pathology was reported as paraganglioma with a central necrotic area of $8 \mathrm{~mm}$ in diameter at the size of $4.1 \times 2 \times 1.8 \mathrm{~cm}$ (Fig. 2). Anti$\mathrm{SDHB}$ markers were positive in the immunohistochemical examination. We could not get an image of the pathology because the technical resources in our hospital were insufficient. Antihypertensive treatment was discontinued when the patient had no hypertension on his first month of follow-up. At the 24-hour urine delivered during the second month of follow-up, the VMA value reached normal levels (5.1 $\mathrm{mg} / 24$ hours). The patient was followed up by thorax CT taken every 6 months for 2 years. At the end of the first and second year, VMA values were checked against the risk of malignancy and recurrence ( $1^{\text {st }}$ year value: $4.9 \mathrm{mg} / 24$ hours, $2^{\text {nd }}$ year value: $3.4 \mathrm{mg} / 24$ hours).

Paragangliomas can be seen with Carney's triad (gastrointestinal stromal tumors (GIST) and pulmonary chondromas) when they are extra-adrenal. The presence of pheochromocytoma may be associated with germline mutations in von Hippel-Lindau (VHL) and neurofibromatosis type 1
(NF1) tumor suppressor genes and the RET proto-oncogene. Family history should be taken regarding VHL syndrome, NF type 1 and multiple endocrine neoplasia type 2 in case of coexistence of pheochromocytoma and paraganglioma [3].

Thoracic and mediastinal paragangliomas are highly vascular tumors, some of which can invade directly into the heart, the main vessels, the trachea, and the vertebral column. It should be kept in mind that bleeding may occur during the operation or during the biopsy procedure. Due to the high blood supply, cases treated with embolization in the literature have been reported [4]. During surgery, first finding and connecting or clipping the main artery and the main vein of the mass reduces the risk of sympathetic discharge that can occur during the manipulation of the mass.

Thorax and abdominal CT are adequate for tumor localization. Considering the frequency of random adrenal masses, patients scheduled for surgery should be evaluated by preoperative scintigraphy of iodine-123-meta-iodobenzyl guanidine (123/-MIBG) or octreotide with indium 111 (111-OCT). The PET-CT examination should also be done for the possibility of malignancy [5]. As in our case, central necrosis can be interpreted as pheochromocytoma detection.

Paragangliomas located in the thorax and mediastinum can be excised with minimally invasive intervention with the help of VATS [6]. $\alpha$ - and $\beta$-Blockers should be available against hypertension and sympathetic hormone discharge during the perioperative period. The opinions of the departments of Cardiology and Endocrinology should be taken before surgery. Patients should be followed up for endocrine disorders and should be examined whether urine VMA value is normalized at the outpatient clinic.

As a result, posterior mediastinal paraganglioma can be detected at the early stage and can be successfully treated with surgery, especially when it is functional. Patients should be carefully prepared and followed up for surgery, hypertensive crisis and cardiac arrhythmias.

\section{Disclosure}

The authors report no conflict of interest.

\section{References}

1. Soomro NH, Zahid AB, Zafar AA. Non-functional paraganglioma of the mediastinum. J Pak Med Assoc 2016; 66: 609-611.

2. Muriana P, Bandiera A, Ciriaco P, Arrigoni G, Negri G, Zannini P. A case of endobronchial paraganglioma. Ann R Coll Surg Engl 2017; 99: 28-30.

3. Shi G, Cui Y, He Y, Gong M. An unusual case of incomplete Carney triad: an 18-year-old girl suffering from multiple benign tumors. J Thorac Dis 2016; 8: 1202-1206.

4. Doğusoy I, Yapıcı F, Çizmeli MO, Demirbağ H, Yıldırım M. Preoperative embolization in the management of a non-functioning mediastinal paraganglioma. Turk Gogus Kalp Dama 2013; 21: 1141-1144.

5. Kedia R, Hansen N, Goldner W. Teaching case: nuclear imaging in metastatic paraganglioma. J Nucl Med Technol 2016; 44: 251-252.

6. Ma L, Mei J, Liu L. Thoracoscopic resection of functional posterior mediastinal paraganglioma: a case report. J Thorac Dis 2014; 6: 1861-1864. 